\title{
Pós-Dradiviano Sateré-Mawé
}

\author{
Parentesco y rituales de afinabiliadad
}

Gabriel O. Alvarez

Prof. PPGAS/FCS/UFG

\section{Resumo}

O material etnográfico Sateré-Mawé foi analisado utilizando-se as diferentes tradições teóricas da antropologia do parentesco. A analise do sistema de parentesco permite preencher um vazio bibliográfico sobre o grupo e também é a porta de entrada para transcender o âmbito dos termos de parentesco e compreender a alteridade. As conclusões teóricas no campo do parentesco trazem uma interpretação do ritual da tucandira, que transcende a exegese nativa e mostra como o ritual transforma, performaticamente, a afinidade virtual em afinidade potencial e os afins potenciais em afins efetivos. A partir desta analise concluímos que o ritual da tucandira como instituição, ordena a circulação de mulheres de forma tão eficaz como outras instituições clássicas do parentesco, como o casamento entre primos cruzados e os sistemas dradivianos.

Palavras-chave: parentesco; ritual; Tupí; Sateré-Mawé; dravidianato amazônico

\section{Abstract}

The Sateré-Mawé ethnografic material was analyzed by the perspective of different theoretical traditions of anthroplogy of kinship. The analysis of Sateré-Mawé kinship sistem allows us to fill a bibliographic lack on this subject, and it also works as an approach that makes possible to transcend the terms of kinship to the comprehension of otherness. The theoretical findings on the field of kinship allows us to interpret the ritual of tucandira, which shows how this ritual performatically transforms the virtual affinity into pottencial affinity and the potential affinity into actual affinity. From the analysis of kinship and ritual, we propose to interpret the ritual of the Tucandira as an institution that arranges the circulations of women as effectively as other usual institutions of kinship, such as marriage beteween cross cousins and dravidians systems.

Keywords: kinship; ritual; Tupí; Sateré-Mawé; amazonic dravidianato 


\section{Resumén}

El material etnográfico Sateré-Mawé fue analizado por la perspectiva de diferentes tradiciones teóricas de antropología del parentesco. El análisis del sistema de parentesco Sateré-Mawé permite cubrir un vacío bibliográfico acerca de la temática y también será la puerta de acceso que permitirá trascender el ámbito de los términos de parentesco para la comprehensión de la alteridad. Las conclusiones teóricas en el campo del parentesco, permiten una interpretación del ritual de la tucandira, que muestra como el ritual transforma performáticamente la afinidad virtual en afinidad potencial y los afines potenciales en afines efectivos. A partir del análisis del parentesco y el ritual, proponemos interpretar el ritual de la Tucandira, como una institución que ordena la circulación de mujeres de forma tan eficaz como otras instituciones clásicas del parentesco como el casamiento entre primos cruzados y los sistemas dradivianos.

Palabras clave: parentesco; ritual; Tupí; Sateré-Mawé; dravidianato amazónico 


\section{Pós-Dradiviano Sateré-Mawé}

Parentesco y rituales de afinabiliadad'

Gabriel O. Alvarez

Prof. PPGAS/FCS/UFG

\section{Los Sateré-Mawé: parentesco, afinidad y estructura social}

Los Sateré-Mawé, presentes en varias crónicas sobre la historia regional, permanecieron en un cono de sombra en la teoría antropológica. Existen pocos trabajos sistemáticos sobre el grupo y ninguno de ellos presenta una descripción satisfactoria de su sistema de parentesco.

En primer lugar tenemos los trabajos pioneros realizados por Nimuendajú (1948) y por Nunes Pereira (1967; 2003). El primero de eses trabajos corresponde a breves visitas de campo realizadas en 1923, cuya información fue reunida para la elaboración del verbete sobre el grupo en el Handbook os South American Indians (Steward 1948). Nimuendajú presenta el grupo, siguiendo el modelo de Steward, caracterizandolo como una sociedad de pequeña escala adaptada a la floresta tropical. El autor señala que el grupo es de lengua Tupí, organizado en clanes y presenta algunos datos sobre tecnología y la economía del grupo, así como también realiza una descripción del Porantin. El autor no aborda el tema del parentesco, por no tener formación académica para trabajar con los datos.

Posteriormente, Nunes Pereira visita el grupo en 1939 y colecta informaciones publicadas originalmente en 1940. Nunes Pereira (2003) realizó un trabajo dentro de los esquemas de la época, en el que analiza: habitación; medios de locomoción; cultivo; actividades económicas; descrive aspectos de algunos rituales y aborda cuestiones sobre religión nativa y los paini (shamanes). En la segunda parte de la obra presenta leyendas y tradiciones

1 Este artículo fue uno de los productos de mi investigación de pós-doctorado en el Programa de PósGradiación en Antropologia Social de la Universidad de Brasília PPGAS/ICS/UnB en el periodo 2003-2007. 
registradas con un interés folcklórico. En el Moronguetá, el autor amplía, un poco, la colección de mitos del grupo (Nunes Pereira, 1967). En esos mitos, los conflictos con los tíos y con los suegros son una constante que atraviesa las diversas narrativas míticas.

En la década de 1950, con el apoyo de Nunes Pereira, el antropólogo americano Seth Leacock realiza su trabajo de campo para su tesis de doctorado (Leacock 1958, apud MacDonald 1965). No tuvimos acceso a ese trabajo. El paradigma dominante en la época era la teoría de la aculturación. En un trabajo publicado en el Boletim do Museu Goeldi, Leacock (1964) analiza el grupo con una perspectiva centrada en el cambio cultural. En este trabajo, en una nota de pié de página, el autor señala que el grupo tiene un sistema de primos de tipo Omaha.

Los autores contemporáneos que trabajaron con el grupo a finales del siglo $\mathrm{XX}$, privilegiaron otros aspectos, como las luchas políticas, salud y cosmología, aspectos míticos, mas no trabajaron el tema de parentesco y organización social (Lorenz 1992, Figueroa 1997).

Existe una discusión sobre parentesco Tupí, que aparece como paño de fondo de las discusiones sobre parentesco en la etnología brasilera. Una de las pocas menciones sobre el parentesco Sateré- Mawé fue realizada por Leacock (1964), y utilizada por MacDonald (1965) en su trabajo sobre parentesco Tupí. Para estos autores, el sistema de parentesco Sateré-Mawé sería del tipo Omaha, una variación del sistema Tupí, que se caracterizaría por la descendencia bilateral, fusión bifurcada, sistema de primos Iroques, casamiento preferencial con primas cruzadas y avunculato (McDonald 1965). Laraia (1972), que realizó trabajo de campo con los Suruí, grupo recién contactado en la época de su investigación, sostiene la hipótesis de que los sistemas de parentesco Tupí se caracterizarían por la descendencia unilinear, patrilinear, residencia patrilocal, con casamiento de primos bilaterales y/o con la hija de la hermana. En el debate contemporáneo, Fausto, sostiene que el sistema Tupí se caracteriza por dos terminologías, por un lado un dradiviano horizontal, con un componente avuncular oblicuo (Fausto, 1995). Por otro lado este debate gana nueva luz a partir de las consideraciones sobre el dravidianato amazónico, elaboradas por Viveiros de Castro $(1995,1996,1999)$.

Las diversas posiciones en este debate, nos remiten a diferentes perspectivas teóricas. A diferencia de otras disciplinas, en la antropología coexisten diversos paradigmas y es posible realizar un análisis de los 
datos desde la perspectiva de cada una de ellas. Consideramos la antropología como un conjunto de tradiciones donde los diferentes paradigmas corresponden a diferentes tradiciones nacionales, estrechamente relacionadas con el desenvolvimiento de la antropología en los países centrales (Cardoso de Oliveira 1988).

A partir de una perspectiva inspirada en la tradición norteamericana, podemos describir el sistema de parentesco Sateré-Mawé como un sistema con fusión bifurcada en la generación ascendente y un sistema de primos análogos al sistema Omaha. La fusión bifurcada se caracteriza por la aplicación del mismo término para el padre $(\mathrm{F})$ y el hermano del padre (FB), y otro término para la madre (M) y la hermana de la madre (MZ), y términos diferentes para la hermana del padre (FZ) y para el hermano de la madre (MB). En el caso de los Sateré-Mawé, tenemos también una diferenciación por senioridad, a partir de la diferencia de edad entre los parientes clasificatorios, tenemos así un grupo de "padres" ' $F$ ' (FB), (identificado por el subfijo colectivo - 'in), con un padre más viejo (FeB) y otros padres más jóvenes (FyB), y una grupo de madres ' $M$ ' (MZ), que se divide en madre mayor (MeZ) y madre menor (MyZ). En la generación ascendente de ego, en el caso de los Sateré-Mawé, se usa el término ui' ywot para padre (F), ui' ywot in (-in "colectivo") para el grupo de padres y las formas -tang para más viejos e -hit para los más jovenes: ui' ywot'tang [ui' ywot + tang > ui' ywo rang] para el padre más viejo (FeB) y ui' ywot' hit [ui' ywot + hit > ui' ywon hit] para el padre más joven (FyB). Uity es el término utilizado para madre (M), uity' in para el grupo de madres (MZ), siendo uity ky' it para la madre más vieja (MeZ) y uity kypy' yt para la madres más joven (MyZ). Un término diferente es utilizado para las hermanas del padre (FZ) y otro para los hermanos de la madre (MB), o sea la bifurcación para las categorías cruzadas. En el caso de la hermana del padre (FZ), la categoría clasificatoria es api $i$ i. Para los hermanos de la madre (MB) el término utilizado es i’kywyt.

Desde el punto de vista de la tradición norteamericana, la generación de ego puede ser descripta como presentando también una fusión bifurcada y una estructura de primos que se aproximaría formalmente al sistema Omaha. En la generación de ego, la categoría utilizada para hermano (B) se aplica también a los hijos de los hermanos del padre (FBS), y a todos los hombres del ywania (clan) (FFBS, etc.). Esta categoría aparece dividida por la aplicación de la diferencia de senioridad entre el hermano mayor y el más joven. En la 
generación de ego, se utiliza el término uheywyt, traducido como "mi hermano" (B, FBS), como una categoría que se aplica a los descendientes de los "padres" (F, FB, FFB,...), uheywyt' in es la categoría colectiva que denomina al grupo de uheywyt ('B'). Por la aplicación del principio de diferenciación jerárquica la categoría se desdobla en los término uheyké et para el hermano mayor (eB) y uheywyt hit para el hermano más jóven. Cuando se aplica para los "hermanos" del ywania, la diferenciación entre los mayores y menores aparece marcada por la aplicación de las formas -tang para el mayor e -hit para el más joven: [uheywyt tang: uheywyt + tang > uheywyt rang] ( $\left.\mathrm{e}^{\prime} \mathrm{B}\right)$ y [uheywyt hit: uheywyt + hit > uheywyn hit] $\left(\mathrm{y}^{\prime} \mathrm{B}^{\prime}\right)$ respectivamente.

Inyt es el término utilizado para hermana (Z), que se aplica también a la hija del hermano del padre (FBD), la hermana clasificatoria. El prefijo posesivo uhe- transforma el término uheinyt en "mi hermana". Las categorías uheinyt yiang para la hermana más vieja (eZ) y uheinyt wap para la hermana más joven (yz). Cabe destacar que la extensión del término inyt para las mujeres del ywania (clan), orienta las alianzas matrimoniales. La prohibición de casamiento con las inyt expresa al nivel de las relaciones lo mismo que la prohibición de endogamia dentro del ywania al nivel de las clases (Dumont, 1975). Las hijas del hermano mayor del padre son también llamadas de ui' ywot tang [ui' ywot + tang si' iwo rang] sak'inyt, o "hija de mi padre mayor" (eFBD). Si ego es mujer, el término uheyké et se aplica al hermano mayor (eB) y uheywyt, para el hermano más joven ( $\mathrm{yB})$, uheiky' it para "mi hermana mayor" (ez) y uikypy' yt para la hermana menor (yz).

En el caso de los primos cruzados matrilaterales, los hijos de los $i^{\prime} k w y t$ son clasificados como imopyt i'kwyt (MBS), (que podría ser traducido como una especie de pariente de la esposa), y para la prima cruzada matrilateral (MBD) se aplica el término imompyt haryporia, donde poria es mujer y haryporia sería una esposa en potencial. La categoría haryporia aparece en los límites de las categorías de parentesco, una vez que el término se aplica a todas las mujeres esposas en potencial, pertenecientes a los otros ywanias (clanes), que no el de ego. Una vez que para esposa se aplica el término hary, y para mujer en general la palabra poria, podemos entender haryporia como esposa en potencial.

La claridad con que son clasificados los primos paralelos, contrasta con la ambigüedad de la clasificación de los primos cruzados. Particularmente llama la atención el contraste entre los primos cruzados matrilaterales, que ocupan una posición clara de conjugues potenciales, o afines, y los primos 
cruzados patrilaterales, colocados en una zona nebulosa, invisibilizada, inclusive para los informantes. Cuando volví del trabajo de campo con las genealogías para sistematizar los datos para construir el sistema de parentesco, mi colega el prof. Roque Laraia me llamó la atención para la ausencia de los términos para los primos cruzados patrilineares. Para la hija de la hermana del padre (FZD) aplican el término api'ap, que presenta gran similitud con el término usado para la hermana del padre (FZ), api i i. A pesar de tener este dato, no tenía ningún registro sobre la categoría empleada con el hijo de la hermana del padre (FZS). Al volver para el trabajo de campo, me propuse resolver ese punto con mis informantes, y me sorprendí con la ausencia de esa categoría. Se estableció un debate sobre la ausencia de esa categoría, que pasó a ser un enigma que finalmente fue resuelto cuando el tuxaua (jefe) señaló que el término para el hijo de la hermana del padre (FZS) sería kurumin sí era niño, y kurun sí era joven y ya pasó por el ritual de la Tucandira.

Pasemos ahora a las categorías de parentesco por afinidad en la generación de ego. La esposa es llamada de ihary (W) y la categoría para la hermana de la esposa (WZ) es iky'it. Para el hermano de la esposa (WB) se utiliza el término $i^{\prime}$ kywyt. Para diferenciar el hermano de la madre (MB) del hermano de la esposa (WB) se aplica el término ity kywyt para el hermano de la madre. El término henany' in es utilizado para la hermana de la madres (WZ).

La generación descendente aparece marcada por el término colectivo membyt ' in, que puede ser traducido como hijos/as (S/D). Para "mis hijos", se utiliza el término imembyt' in ('S'/'D', S, BS, FBS, D, BD, FBD). Se utiliza el término imembyt 'in hà (eD) para la hija mayor. El uso de este término es extensivo a los hijos de los hermanos, como por ejemplo, los hijos de las hermanas (inyt' in) son llamados de inyt membyt' in (ZS); los hijos de hermano más viejo uheykéet membyt in (eBS) y para los hijos del hermano más joven se usa el término uheywyt membyt' in (yBS). Los hijos de la hermana mayor de la esposa, iki' it, reciben el término iki' it membyt 'in (WeZS), y los hijos de la hermana menor de la esposa ikypyt' $y t$, reciben el término de ikypyt' yt membyt ' in (WyZS). En todo este grupo de términos, la diferenciación entre los membyt remite a la generación ascendente.

Dumont, $(1875,1983)$ señala que los sistemas de parentesco del tipo de fusión bifurcada, se fusionan en la generación $+\mid-2 y$ se bifurcan en las generaciones centrales $(G$ o y $G+\mid-1)$. En el sistema Sateré-Mawé encontramos esta fusión en la segunda generación descendente, donde se aplican los términos 
uhemiarira para las nietas y uhemiariru para los nietos. La fusión no es completa en la segunda generación ascendente, en la que se aplica el término ase' $i$, para el padres de la madre (MF); hary para la madre de la madre (MM) y para la madre del padre (FM). Un término diferente, uiywot aséi se aplica al padre del padre (FF). Esa categoría marca la diferencia entre la linaje patrilinear y los parientes por afinidad, y refuerza la patrilinearidad al tratar FF como un hermano jerarquicamente más viejo, recordando que la categoría ywot es aplicado al grupo de siblings que incluye al F y FB.

Desde el punto de vista de la tradición formalista norteamericana, se puede describir el sistema de parentesco Sateré-Mawé como un sistema con fusión bifurcada en la generación ascendente, o sea, con un término clasificatorio para el padre y los hermanos del padre y un término clasificatorio para la madre y las hermanas de la madre. Tanto el término para los hermanos del padre, como el término para las hermanas de la madre tienen una forma plural y una diferenciación de senioridad entre los mayores y menores. La misma categoría que se aplica al padre se aplica para el hermano del padre y para el padre del padre, que puede ser representado por la equivalencia $\mathrm{F}=\mathrm{FB}=\mathrm{FFB}$.

El trazo distintivo del sistema de parentesco Sateré-Mawé es la categoría $i^{\prime} k y w y t$, una categoría que expresa la relación de parentesco por afinidad y que se aplica a los parientes afines masculinos de diversas generaciones $(\mathrm{MB}=\mathrm{MBS}=\mathrm{WB})$. Esas equivalencias llevaron a Leacock (1964) a clasificar al sistema Sateré-Mawé como teniendo un sistema de primos del tipo Omaha. Esta estructura formal, con todo, no corresponde a los sistemas de tipo Omaha de los grupos Jé de la región Centro-Oeste de Brasil, donde la estructura está asociada a la transmisión de nombres, obligaciones rituales y una figura de paraparentesco como el amigo formal. Desde el punto de vista de Fausto (1995), este sería el componente oblicuo que podría ser el reflejo terminológico del avunculato Tupí. Para Laraia (1972) sería un índice que indicaría la presencia de grupos de descendencia unilinear diferentes.

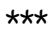

Desde el punto de vista de la tradición Británica de antropología social, la perspectiva teórica estaría orientada para ver el parentesco como base de la estructura social. El parentesco define la relación de pertenencia al grupo, al clan, y se manifiesta en los sistemas de actitudes orientados por el 
parentesco. Esta tradición se inició con el método genealógico de Rivers, que permitía visibilizar las redes de relaciones sociales del parentesco, y nos permite ver el sistema de parentesco como una red que opera al nivel de una micro-política, sobre todo en las sociedades sin Estado.

La pertenencia política al grupo se dá por el parentesco. Así como otros grupos Tupí, los Sateré-Mawé son patrilineares al punto de no considerar como parientes aquellos vinculados por la linaje materna (Laraia 1972; Fernandes 1989), o considerarlos un tipo de parientes diferente. En el caso de los Sateré-Mawé, durante el trabajo de campo levanté relatos que apuntan en ese sentido: "El hombre trae los hijos e hijas en la sangre, así es que decían los antiguos, en la sangre están los hombres y mujeres que Dios coloco ahí, tantas personas vos vas a traer: uno, dos, tres hijos, miariru (netos)" (João Mathias, 76 años, Molongotuba). El parentesco consanguíneo patrilinear determina la pertenencia al grupo. La aplicación de las categorías de parentesco y su extensión al nivel de los clanes, crean una red micro-política que orienta las actitudes con los que son de dentro del grupo y los de afuera. Las categorías para los consaguíneos $(\mathrm{F} / \mathrm{B} / \mathrm{Z})$ se proyecta al nivel del ywania (clan). Esta relación de pertenencia a los ywania, como unidades separadas dentro del grupo, se hace evidente cuando los nativos traducen el término como "nación". Las categorías de parentesco que se aplican a los consanguíneos sirven de modelo para regular las relaciones al interior del ywania. En la generación de ego, la categoría de parentesco uheywyt (B) que se aplica también a los FBS, así como sus variantes jerarquicas uheywyt tang [uheywyt + tang > uheywyt rang] para el hermano mayor ( $\left.\mathrm{e}^{\prime} \mathrm{B}^{\prime}\right)$ y uheywyt hit [uheywyt + hit > uheywyn hit] para el hermano menos ( $\left.\mathrm{y}^{\prime} \mathrm{B}^{\prime}\right)$ se proyectan al nivel del ywania. De forma análoga, la categoría para inyt (Z) se proyecta al nivel del ywania. Las mujeres del ywania son llamadas de inyt 'in, y la prohibición del casamiento entre hermanos orienta la elección de los conjugues posibles. La extensión de los términos de parentesco, y la expresión descriptiva en ocasiones específicas sirve para diferenciar el parentesco de sangre y el parentesco en el ywania.

Los Sateré-Mawé poseen grupos de descendencia unilineal y una organización mínima en clanes o ywanias. Tal como afirman diversos autores, se trata de clanes no localizados, exogámicos, patrilineares y patrilocales, con espacio para la manipulación de las reglas de residencia. El grupo residencial está compuesto por la família extensa, el grupo de siblings aparece como una unidade que organiza el sistema de actitudes del grupo. Cabe destacar que 
en la generación de ego, las categorías de consanguinidad se proyectan en la organización en clanes, de forma que los hombres y mujeres del clan son considerados como hermanos y hermanas. La diferencia entre hermano mayor y hermano menor organiza el sistema de senioridad al interior del grupo de siblings que se reproduce al nivel del ywania. Se espera del hermano mayor una actitud de líder y de consejero con los menores. El grupo de siblings también es movilizado en el caso de venganzas de sangre o por acusaciones de brujería. En la cosmología que aparece reflejada en los mitos y en los "cantos de guerra" del ritual de la Tucandira, los ywania luchaban separados, hasta que el ywania Sateré organizó la alianza entre los clanes para luchar contra el ywania de los enemigos caníbales, los meirú.

El sistema de parentesco opera a partir de diferentes dimensiones: generacional, edad relativa, sexo y parentesco por afinidad. Las dimensiones generacional y la edad relativa ordenan la terminología de parentesco por consanguinidad. El parentesco ordena también, al nivel del sistema de actitudes, las relaciones de senioridad con los mayores. En la tradición Sateré-Maé se espera que el hermano mayor asuma una actitud de líder, que encabece las iniciativas y que oriente a los mas jóvenes. La relación de senioridad mayor/ menor orienta las relaciones sociales. Durante el trabajo de campo, cierto día un Sateré me confió que él, como hermano mayor sentía falta de tener un hermano mayor que lo aconsejase, por eso el recurría al hijo mayor de su "padre mayor" (FeBeS).

Desde el punto de vista de esta tradición teórica, también conocida como teoría de la descendencia, se resalta el papel del sistema de parentesco en la organización social del grupo. La estructura pode ser caracterizada como un grupo con clanes no localizados, donde el grupo de siblings tiene un peso significativo, a pesar de no actuar como grupo corporado. Los clanes no tienen prerrogativas rituales, a pesar de esto tienen un peso importante en la elección de los cónyugues.

Las reglas de patrilocalidad no se aplican de forma rígida como en las sociedades trabajadas por los africanistas. La economía política de los cuerpos crea un espacio para la manipulación de la regla. Una de las estrategias disponibles para el incremento de las aldeas es la incorporación de los yernos (Viveiros de Castro, 1986: 418, Rivière, 2001: 127ss). Como en otros grupos de la región amazónica, los tuxauas tienen mayores chances de atraer un yerno para vivir con su hija, incorporándolos a la familia extensa hasta que tengan 
hijos y creen una nueva unidad domestica. Esta estrategia estimula el crecimiento de la aldea y la diversificación del número de clanes. En la cosmología del grupo el ideal de que las aldeas sean compuestas de varios ywania, porque son diferentes tipos de gente, con cualidades diferentes.

La relación política de pertenencia al grupo se da en primer lugar por la descendencia patrilinear. El parentesco patrilineal define la posición en el sistema de clanes. Las personas pertenecen al clan de su padre, del padre de su padre, y por pertenecer al clan, pertenecen al grupo Sateré-Mawé. Los que son hijos de madre Sateré-Mawé y padre no-sateré son considerados sateré asiag, mestizos, por contraste con los sateré sesse, puros o verdaderos. La relación de pertenencia al grupo se da por la descendencia, mas existen otra forma de incorporación, por el casamiento con las mujeres Sateré-Mawé. En estos casos, los hijos de estas mujeres son considerados sateré asiag, o impuros, y esa condición de mestizo se transmite de generación en generación. En una de las genealogías, registré la transmisión patrilineal de esa condición por cinco generaciones, en otros casos, se levantaba esa sospecha, remontándola a la época de la guerra con los Mundurukú, enemigos históricos del grupo. Registré varios casos de mujeres Sateré casadas con mestizos, karaiwas (blancos) y casos de mujeres casadas con mestizos indígenas. Durante el trabajo de campo, en la aldea había dos casos de indios mestizos, Ticuna e Hixkariana, casados con mujeres Sateré. En mi último viaje, eses mestizos ja habían partido de la comunidad.

Uno de los tuxauas me comentó que, cuando una mujer del grupo se casa con un karaiwa, y lo lleva a vivir en la aldea, ellos le dan consejos. "Sí él no acepta los consejos, entonces es expulsado y tiene que irse -afirmó el tuxaua y, con un brillo en los ojos completá la frase- y quedamos con más mujeres".

Desde la perspectiva de la tradición francesa, conocida como teoría da la alianza, el análisis estaría centrado en el intercambio, en la circulación de mujeres y en el relacionamiento entre los clanes, con base en el casamiento entre primos cruzados. La terminología de parentesco Sateré-Mawé es compatible con el casamiento con la prima cruzada matrilateral y con el avunculato. Esta correspondencia terminológica puede haber llevado a Leackoc (1964) a realizar la afirmación de que en el pasado existía casamiento entre 
primos cruzados. Si tal casamiento ocurriese de hecho, estaríamos frente a un esquema clásico de intercambio generalizado (con un componente oblicuo avuncular). Por otro lado, si el sistema fuese Omaha, tal como sostenía el mencionado autor, no se encajaría en las estructuras elementales de parentesco y sería del tipo semi-complejo (Leví-Strauss, 1967; 1982). En las genealogías que levanté en el trabajo de campo y en las preguntas a los informantes no conseguí observar casos de casamientos entre primos ni avunculato, a pesar de la remota mención a algún caso en alguna otra aldea. Uno de los corolarios de la teoría de Leví-Strauss (1982) es que la mujer (o la circulación de mujeres por el casamiento) es responsable por la alianza entre los grupos. Como afirma Balandier (1977), en su interpretación más dinámica de esta teoría, la mujer es la responsable por la alianza, mas es una alianza inestable y tensa que puede ser quebrada a cualquier momento. La mujer ocupa el lugar ambiguo de fiadora de la alianza, mas también puede quebrarla.

Una de las críticas de Dumont (1975) a la teoría estructuralista de Las estructuras elementales de parentesco (Leví-Straus, 1982), es que el autor no es claro, y oscila entre el nivel de las relaciones y el nivel de las clases. No es lo mismo casarse con la hija del hermano de la madres, que casarse con alguien a quien se le aplica esa categoría clasificatoria. En uno de los casos se opera a nivel de relaciones, en el otro a nivel de las clases. En su artículo sobre el dradiviano, Dumont (1983), señala que la alianza no es entre el hombre y la mujer, y si entre dos grupos de parientes afines del mismo sexo, mediados por el vínculo del casamiento, y remite la alianza para la generación ascendente. Lo relevante, para este autor, es la alianza entre F y MB, que opone ego y F a los MB. La afinidad no es producto del casamiento, las categorías de afinidad preceden a la alianza matrimonial. Una de las características del dradiviano es la reproducción de las categorías de parientes afines y consanguíneos, creando alianzas diacrónicas o genealógicas (Dumont 1983). En las genealogías levantadas durante el trabajo de campo junto a los ancianos, apareció la repetición de alianzas matrimoniales entre los mismos clanes en las tres generaciones ascendentes. No ocurre lo mismo en las generaciones de los hijos $\mathrm{y}$ nietos de estos ancianos.

$\mathrm{Al}$ analizar los sistemas a la luz del modelo dradiviano clásico, cabe destacar la posición ambigua de la hermana del padre. Dumont clasifica a la hermana del padre (FZ) como un pariente por afinidad, una vez que casada irá a vivir en la casa del conjugue. Por otro lado, como afirma Viveiros de Castro 
(1996), en las tierras bajas existe todo un juego político en que la regla de la patrilocalidad puede ser manipulada en función del prestigio político del suegro, en este caso el yerno pasa a vivir con los parientes de la mujer. Otra alternativa es que la mujer vuelva a vivir con sus parientes consanguíneos después de la separación del conjugue, en estos casos es común que la mujer vuelva a la aldea de los padres. Como señala Viveiros de Castro (2002: 48), en esas situaciones se aplican tecnonimias que enfatizan el parentesco agnático en detrimento de las categorías de afinidad. En estos casos se aplica la categoría ui' ywot inyt, o literalmente "hermana de mi padre".

Un cuestión que queda abierta es si la madre es una categoría consanguínea o colateral. Según Shapiro (in Viveiros de Castro 1996), la clasificación de la madre como consanguínea o colateral, sería uno de los trazos diagnóstico que permitirían diferenciar entre dos variantes de la fusión bifurcada, la dradiviana y la kariera. El sistema kariera está organizado a partir de clases matrimoniales y el dradiviano, en su versión hindú, tiene como base la clasificación de los parientes en las categorías de consanguíneos y afines. En el dravidianato amazónico, aplicado a sociedades cognáticas y endogámicas, la clasificación depende de la localización en la estructura y la transformación es una de las reglas del sistema. Si se puede clasificar a la madre como colateral, como se desprende del princípio de patrilinealidad Tupí, en los SateréMawe, el sistema estaría próximo del kariera, pero no existen clases matrimoniales y las alianzas están orientadas por los clanes, ywanias.

Retomando el sistema de términos aplicado a los primos cruzados, cabe resaltar también que, en los casos de alianza simétrica con casamiento entre primos cruzados bilaterales, los términos aplicados a los primos cruzados matrilaterales y patrilaterales coinciden. La otra explicación para la falta de clareza para para los términos para los primos cruzados patrilaterales, podría ser la preponderancia del uso de los términos aplicado a los primos matrilaterales en el caso de un hipotético casamientos de primos bilaterales. Pero en este caso, tal desmembramiento del sistema de primos colocaría también, lado a lado, las categorías de kurum y haryporia.

En el caso de los primos cruzados, en particular el FZS y MBD, kurm y haryporia, parecen términos ubicados fuera de la esfera del parentesco. Estos términos son introducidos en el sistema de parentesco, uno de ellos señalando la esposa en potencial y el otro un no-pariente, que puede transformarse en pariente. Si existiese (en el pasado, en la teoría o en el plano virtual) una 
formula de casamiento con la prima cruzada matrilateral, el kurum sería un yerno en potencial, mas que necesita se afinizado. A partir de la posición del kurum, ego será un i’kywit.

Con el cambio de perspectiva de Leví-Strauss para Dumont, el énfasis pasa de la circulación de mujeres para la relación con los parientes afines, en el caso de los Sateré-Mawé, englobados en la categoría $i^{\prime} k w y t$. La categoría $i^{\prime} k w y t$ tiene que se comprendida como afinidad virtual. La categoría de $i^{\prime}$ kwyt se aplica para el hermano de la madre (MB), para el hijo del hermano de la madre (MBS), para el hermano de la esposa (WB). Fué este término que llevó a Leacock (1964) y MacDonald (1965) a clasificar el parentesco SateréMawé como del tipo Omaha y sería el "componente oblicuo" señalado por Fausto (1995) para el caso Parakaná. Cuando le preguntamos a uno de los tuxauas sobre el significado de la categoría i’kwyt, él respondió: "son los pariente de ella", lo que revela el contenido de afinidad virtual (o potencial) del término. Sí los sistemas dradivianos (Dumont 1983) se caracterizan como aquellos que diferencian los parientes en las categorías de consanguíneos y afines, tenemos en $i^{\prime} k w y t$ el término que expresa esa afinidad como categoría que incluye a los hombres relacionados por medio de la alianza matrimonial: suegros, yernos y cuñados. En la próxima sección volveremos sobre esta categoría de pura afinidad y exploraremos algunas de sus implicaciones para la cosmología Sateré-Mawé.

Este punto es congruente con las preocupaciones de Rivière, que trabaja la afinidad como una relación que puede ser descompuesta en cuatro tipos de relación: conyugal, la matrimonialidad, la afinidad y la afinabilidad ( Rivière 2001:83). La matrimonialidad se caracteriza como una relación que se establece únicamente entre aquellos que pertenecen a sexos opuestos; la relación de afinidad se dá por los parientes próximos de la esposa, sean del mismo sexo o del sexo opuesto. Rivière trabaja la categoría de afinabilidad, como la de aquellos que pueden transformarse en afines efectivos. El autor diferencia también los afines cognatos de los afines no-parientes. Así como el casamiento es la efectivación de la matrimonialidad, la afinabilidad tiene que ser efectivada para la transformación de estos afines virtuales en afines efectivos.

Como señalamos anteriormente, haryporia, que se aplica para la MBD, se aplica también a la mujeres de los otros ywania, pasando del nivel de las relaciones para el nivel de las clases en la determinación de los conjugues posibles. Con esta transformación se pasa de la alianza con un grupo de afines, 
para la potencialización de la afinidad con los diversos ywanias. Por otro lado, la aplicación del término kurum, que tiene como recíproco i’kywit, revela que esa afinidad potencial o afinabilidad tiene que ser efectivada para transformar el kurum en afín. La torsión del esquema dradiviano, formulada por Viveiros de Castro (1996), al transformar las cajas en círculos concéntricos, tiene como una de sus propiedades transformar la alianza bilateral en alianza multi-bilateral. Esa propiedad permite una mayor diversificación del número de alianzas.

\section{Parentesco Tupí y dravidianato}

Para MacDonald (1965:18), el sistema de parentesco Tupí estaría caracterizado por la terminología de parentesco de tipo fusión bifurcada, en la primera generación ascendente y por el sistema de primos del tipo iroques en la generación de ego. Las reglas matrimoniales serían el matrimonio preferencial con una o ambas primas cruzadas, también avunculato, casamiento con la hija de la hermana. Para MacDonald, la regla de descendencia es bilateral, en ambas lineas, paterna y materna que eran reconocidas por ego. Entretanto, el autor reconoce una tendencia fuerte hacia el lado patrilinear en el caso de los Sateré-Mawé, con clanes, residencia patrilocal y familia extensa patrilateral.

El modelo presentado por MacDonald (1965), enfatiza aspectos formales, como la terminología con fusión bifurcada en la generación ascendentes y en la primera generación descendentes; el sistema de primos del tipo Iroqués, de las cuales, Crow y Omaha, constituirían variaciones de la misma estructura de parentesco bilateral. MacDonald concluye por la inexistencia de divisiones unilineares exogámicas. La excepción de los Mundurukú es explicada por la duda en cuanto a su filiación lingüística Tupí. Laraia (1972) se empeña en demostrar la posibilidad de segmentos de descendencia unilinear en las sociedades Tupí, lo que explicaría el casamiento de primos bilaterales.

Laraia (1972), criticó a MacDonald, por el énfasis en el sistema terminológico, y la ausencia del sistema de actitudes que acompaña al parentesco como sistema de organización social. El autor también señala que la existencia de términos de parentesco para los parientes bilaterales, no significa que el sistema sea bilateral una vez que grupos de descendencia unilateral utilizan términos de parentesco con sus afines. MacDonald no llevó en cuenta el sistema de actitudes y la importancia del grupo de siblings como elemento de 
organización social. Cabe destacar que ente los tupis, los sistemas de términos aplicados a los grupos de siblings acostumbran diferenciar entre los hermanos mayores y menores, y que esta diferenciación se corresponde con actitudes diferentes. Para Laraia (1972), inspirado en las teorías de la antropología social británica, el trabajo de MacDonald es meramente clasificatorio. No podemos inferir la organización social de los grupos tupí con el conocimiento del sistema de términos utilizados con los primos o con los términos para la primera generación ascendente y sus reglas de descendencia.

Uno de los puntos centrales del análisis de Laraia es la crítica a la negación de los grupos de descendencia unilineares. Laraia (1972) analiza las reglas de residencia, descendencia, y los sistemas de relaciones para mostrar la posibilidad de existencia de grupos de descendencia unilinear. El autor señala que la filiación, al contrario de la descendencia, es universalmente bilateral. Jurídicamente, solo una parte es capaz de determinar la descendencia; el concepto de filiación complementar (Fortes 1975), surge para establecer los lazos entre el grupo de sibling y los parientes que no transmiten descendencia. En su crítica a MacDonald, Laraia señala la necesidad de ver la definición del grupo sobre "pariente" (consanguíneo). Cita inclusive el ejemplo levantado junto a los Tupinambá por el padre Anchieta, para señalar que para los Tupí el parentesco proviene exclusivamente de la liña paterna: "padres son agentes, madres solo sacos" (Laraia, 1972: 87).

Para Laraia (1972), por los sistemas de los Tupinambá históricos, teniendo un ego masculino, las categorías de parentesco que pertenecen a la sección de ego, no se confunden con las categorías de la sección complementar y poseen una nítida distinción entre generaciones. En la otra sección, las categorías incluyen personas de diferentes generaciones. La regla de matrimonio preferencial de primos bilaterales era la más plausible en un sistema de mitades. Entre los pueblos Tupí incluidos en el análisis, presentaban existencia de clanes los Parintintin, Munduruku y Maués (Sateré-Mawé). En su trabajo junto a los Suruí, Laraia había encontrado cinco grupos de descendencia unilinear, y las genealogías indicaban dos grupos más, extinguidos.

En resumen, para Laraia, (1972:122), el sistema Tupí se caracteriza por la fusión bifurcada en la primera generación ascendente y términos de primos de tipo Iroques en la generación de ego; la dinámica del sistema estaría dada por la descendencia patrilinear, la residencia patrilocal y el matrimonio preferencial con las primas cruzadas bilaterales y con la hija de la hermana, avunculato. 
La búsqueda por linajes era una preocupación históricamente fechada. Trabajos posteriores en las tierras bajas mostraron sistemas de parentesco, con casamiento de primos cruzados, sin la necesidad de clanes o linajes unilineares, como por ejemplo los sistemas dradivianos de la región amazónica, que diferencian los parientes en dos categorías: consanguíneos y afines. La importancia de las relaciones de afinidad en las tierras bajas de América del Sur es un tema abordado por diferentes autores que intentaron aplicar la estructura de parentesco dradiviano en grupos indígenas amazónicos (Rivière 1969, 2001; Overing Kaplan 1984; Viveiros de Castro 2002).

Fausto (1995), en su análisis del sistema de parentesco Parakaná concluye que el sistema Tupí resulta de la articulación de dos estructuras terminológico-matrimoniales. Por un lado una terminología dradiviana, horizontal, y por otro lado una avuncular oblicua. El primer modelo separa/categoriza el grupo a partir de la división entre consanguíneos y afines. El segundo resulta de de la interferencia entre la norma avuncular y la dradiviana (Fausto, 1995: 62). Tanto la prima cruzada, como la hija de la hermana serían una especie de esposa en potencial y los tíos maternos podrían ser clasificados como suegros potenciales y/o cuñados.

El dradiviano de Dumont (1983) se transforma en el dravidianato amazónico en la formulación de Viveiros de Castro. En "Ambos los tres", Viveiros de Castro (1996) analiza las diferentes variantes de la fusión bifurcada: iroques, dradiviano y kariera. Después de presentar las discusiones que reflejan el estado del arte, el autor formula su modelo general que permite explicar las variaciones amerindias de la fusión bifurcada. Al analizar el modelo dradiviano de Dumont (en sociedades patrilineales, matrilineales, patrilocales y uroxilocales), Viveiros de Castro, apoyándose en el análisis de Rivière (1969), Overing Kaplan $(1972,1973,1975)$ y Basso $(1970,1975)$, aplica la noción de dradiviano a sociedades amazónicas, de pequeño porte, con parentesco cognático y casamiento preferencial endogámico con primos cruzados. A diferencia del modelo de Dumont, que propone una organización en mitades bilaterales (consanguíneos y afines), Viveiros de Castro propone un ordenamiento concéntrico, con los consanguíneos en el centro, un circulo externo formado por los por los parientes por afinidad, y un exterior formado por los enemigos. Esta transformación del modelo dradiviano, modifica también el sistema de alianzas, que pasa de un sistema bilateral, para un sistema multi-bilateral (Viveiros de Castro 1996). 
Este esquema, inspirado en Levi-Strauss (1967) es animado por la lógica jerárquica del propio Dumont (1992). En el dradiviano, Dumont (1983) usó su concepto de jerarquía para señalar la diferencia entre el parentesco occidental y el parentesco dradiviano. En el sistema occidental, los consanguíneos ocupan la posición jerárquica de englobantes y los afines, englobados, son transformados en consanguíneos en unas pocas generaciones. Por contraste, en el sistema hindú, afines y consanguíneos son dos categorías que se reproducen en el tiempo. Los sistemas dradivianos producen alianzas que se reproducen de generación en generación.

Viveiros de Castro (2002: 134ss) aplica la noción de jerarquía en la relación entre afines y consanguíneos en su esquema concéntrico. En el círculo interior, la consanguinidad ocupa una posición jerárquica englobante. En este espacio social, que puede coincidir con el de la aldea, los parientes afines son consanguinizados, sea por las actitudes, sea por la aplicación de tecnonimias que enfatizan las relaciones cognáticas, sea por medio de prácticas rituales. En ese modelo socio-espacial, el anillo externo está formado por los parientes afines, los consanguíneos que viven en otras localidades y que se encajan en ese espacio social, son afinizados. La verdadera riqueza del modelo no está en su proyección espacial y si en su proyección cosmológica. A final de cuentas: ¿qué significa un pariente por afinidad en estas sociedades amazónicas? Para responder esta pregunta, Viveiros de Castro (2002: 416) proyecta la categoría de afinidad para la cosmología amazónica y crea la noción de afinidad virtual, inspirado en Deleuze.

Viveiros de Castro (2002: 128) propone la diferenciación entre: la afinidad efectiva o actual (cuñados y yernos), la afinidad virtual (primos cruzado y hermano de la madre), y la afinidad potencial, (cognatos distantes y no-cognatos). A diferencia de occidente, donde las figuras consanguíneas ocupan un lugar central (la figura del padre, de la madre), en las cosmologías amazónicas los parientes afines ocupan el lugar de referencia. Son recurrentes las imagenes del suegro caníbal y los enfrentamientos con los cuñados. Para las cosmologías amazónicas, la afinidad es una condición del parentesco. La afinidad potencial se configura como una categoría virtual, desvinculada del parentesco, la afinidad real solo es efectivada después de la "afinización”, después de la transformación del afín virtual en afín concreto.

Ese segundo circulo, de los parientes por afinidad, está contenido por la categoría de los enemigos. Esa categoría ocupa un lugar central en la filosofía 
amazónica y se vincula con el canibalismo y la predación. El enemigo aparece como alteridad que puede ser incorporada. La figura del enemigo aparece en los rituales Tupinambá, tanto en los rituales de nominación, donde la muerte del enemigo daba derecho a un nuevo nombre y cambio de status, como en los largos rituales en que el enemigo capturado es afinizado y después es sacrificado y canibalizado. Este proceso, interpretado a la luz del parentesco revela que las mujeres no son intercambiadas por otros bienes y símbolos, el intercambio es de cuerpos y solo se aceptan cuerpos como contra-prestación. En los casamientos tradicionales, una de las partes era donadora de mujeres y por ser donadora se transformaba en acreedora. La contra-prestación de la donación explica los casos de avunculato, donde el hombre intercambia la hermana y recibe la hija de esta como esposa. El prisionero recibía la mujer, muchas veces pariente consanguíneo de su captor, y por no tener como responder a la contra-prestación era canibalizado. Un cuerpo sólo puede ser intercambiado por otro cuerpo, en este caso el del cuñado enemigo (Viveiros de Castro 2002: 174). Ese canibalismo Tupinambá fue proyectado para el plano de la cosmología en diversos grupos Tupí. Por otro lado, el autor relaciona el canibalismo con la predación en la caza (Viveiros de Castro 2002: 267).

Este camino nos lleva para el prespectivismo y las filosofías nativas amazónicas. En primer lugar, Viveiros de Castro (2002) contrapuso a la separación entre naturaleza y cultura un esquema ternario, que incluye lo sobrenatural. Desde el punto de vista nativo, la ecuación entre naturaleza y cultura es desdoblada. Si para occidente la cultura se separa y engloba la naturaleza, desde el punto de vista amazónico, es la cultura que se transforma en naturaleza. Son comunes, en la región, las cosmologías que se inician en un tiempo en que "los animales eran como gente" hasta ser transformados en animales. La transformación es otra noción central en los sistemas amazónicos, como lo ejemplifica la transformación del shaman en jaguar. Entre los Araweté, trabajados por el autor, a los ojos de los dioses, los humanos son vistos como el jaguar ve a su victima, o como el cazador ve a los animales. Viveiros de Castro (2002) llama la atención para la importancia cultural que tiene la caza, esto se hace evidente en estos pueblos que teniendo agricultura, realizaron una opción cultural por la caza. Otro índice son los rituales a los cuales se someten los cazadores, tanto antes de emprender la cacería, como a su regreso, así como los tabúes frente a la mujer menstruada, o la costumbre difundida -según el autor- de que el cazador no come su caza. La dinámica cosmológica de estos 
pueblos está centrada en la afinidad como categoría cosmológica y en la predación, la caza. La transformación es una regla del sistema.

Shapiro (1984: 26), al realizar una discusión sobre una definición para casamiento (marriage) en las tierras bajas de América del Sur llama la atención para la ausencia de una abordaje performática. La autora deja abierta la posibilidad de que ese fenómeno se deba al hecho de que los pueblos de la región no acostumbran realizar rituales de casamiento, por contraste con los elaborados rituales funerarios. La diferencia entre parientes afines y consanguíneos ordena diversos rituales, como, por ejemplo, los rituales funerarios entre los PakaaNova (Vilaça 1993). Rivière (2001: 86ss) llama la atención para las relaciones de afinabilidad, las relaciones con parientes afines que no pasan por la matrimonialidad. El define la afinabilidad como la relación entre dos grupos de hombres por medio de las mujeres. Viveiros de Castro (2002), con su esquema concentrico, afirma que es posible el pasaje por las diferentes esferas por medio de rituales. A pesar de realizar esta afirmación, no describe ni indica ningún ritual de este tipo. A continuación, analizaré el ritual de la Tucandira centrado en el papel desempeñado por los afines y los efectos ilocucionarios del ritual.

\section{El waumat a la luz del parentesco y el parentesco a la luz del ritual}

"Anualmente de la casa del jefe -maloca- parte la señal de la fiesta, que, repercutiendo de casa en casa, va de Pará al Amazonas. A esa señal, se prepara caxirí y tarubá, bebida embriagante que anima la fiesta y comienzan las cacerías; moqueada, la caza es guardada para los días de fiesta, que es esperada por otra señal. Cuando se escucha esta, de todas las malocas parten los neófitos, y las doncellas con sus padres, cargados de caza, potes de bebida y de tucandiras se dirigen para la casa-grande del tuxaua" (Nunes Pereira, 2003: 63, traducción del autor)

Los Sateré-Mawé afirman que actualmente no hay una fecha cierta para realizar el ritual de la Tucandira. Presencié mi primer waumat en septiembre, realizaron otro durante mi permanencia en la aldea, y presencié dos más que ocurrieron en el cierre de asambleas. Mi primera descripción del ritual fue superficial y apoyada en la observación directa durante un trabajo de consultor para el Ministerio de Previsión Social (Alvarez, 2002). En esa oportunidad realicé una descripción de las fases del ritual, a partir de las informaciones 
recibidas por mis informantes, me concentré en el ritual de la Tucandira como en un rito de pasaje para los jóvenes (Van Gennep 1978). Uno de los datos relevantes para esa investigación era que los jubilados del grupo habían colaborado con su dinero en la compra de alimentos para la realización del ritual.

En otro trabajo describí el ritual a partir de la exegesis nativa, apoyándome en las interpretaciones de diferentes painis (shamanes) y cantadores (Alvarez, 2007). El ritual era visto por los nativos como parte de la construcción de los cuerpos, a imagen de la salud preventiva; como alegoría del coraje simbolizada por las penas del halcón-real; enfatiza la tucandira como alegoría de la mujer. Esa interpretación, apoyada en la exegesis nativa permitió una interpretación más profunda, pero presa a las interpretaciones conscientes. En esta sección pretendo complementar esa interpretación a la luz de las consideraciones teóricas que se desprenden del análisis del sistema de parentesco Sateré-Mawé, realizadas en las secciones anteriores del trabajo.

Tambiah (1985) sugiere que el ritual puede ser analizado como performance en tres sentidos: como aparato comunicacional multi-medios, que entrelaza comunicación verbal y no verbal; en el sentido de tercero referencial de Pierce, cuando los símbolos nos remiten a un tercero que los baña de sentido; y performático, en el sentido de Austin (1997), cuando se hacen cosas con palabras, y con símbolos. Creo que en ese trabajo (Alvarez 2007) realicé un buen análisis del ritual como performance, a pesar de esto quedaron algunos puntos en abierto, que serán retomados aquí a la luz del parentesco: ¿Cuales son los efectos ilocucionarios del ritual de la Tucandira?

Los rituales operan por medio de símbolos, y el símbolo más importante del ritual es la tucandira como alegoría de la mujer. Esa representación de la mujer mítica esta cargada de sentido a partir de la cosmología del grupo. La presencia de la representación de la mujer como símbolo multifacetado despertó mi interés, y no quedó totalmente resuelto en mi interpretación anterior del ritual de la tucandira, en gran parte preso a las interpretaciones dadas por los informantes.

En la interpretación nativa del ritual, la tucandira es una alegoría de la mujer. Esa haryporia/tucandira, tirada de tres especies diferentes de árboles, tiene diferentes cualidades. Las interpretaciones levantadas son que serían también los tres personajes míticos femeninos: Uniawasap, madre del guaraná; otra de las mujeres sería la que acompaña a Anumará hit (héroe cultural mítico), y la tercera sería moi-ria, un tipo de cobra, también alegoría de la 
transformación. Esa tercer mujer, llamada de Onamãkuru’i (Figueroa 1997: 367) o Moiria int sakuri en mis registros, y es la mujer con que fue hecha la tierra, es ella quien quedo con la cara volteada para la tierra, que llama a los hombres para hacerle compañía. Sería Unhanmangarú en Nunes Pereira (2003: 111) y representa la alegoría de la muerte. En la explicación del painí(shaman):

- "Moiria int sakurí es una mujer negra. La otra mujer es una mujer pintada, como el jaguar, y la otra mujer es blanca. Moiria, quiere decir un animal que se transforma, en mujer o en cualquier otra cosa. No es la serpiente, sino un tipo de moi (serpiente) que se transforma. Entonces la historia es así, la mujer negra es la que no tiene paciencia, la que se transforma. Por ejemplo, cuando uno está alterado con otro, que pasa y ya queda con rabia de esa persona. Entonces, esa mujer/serpiente moiria, ella es muy alterada con cualquier tipo de persona" (Atenor, Molongotuba, 2004).

Debo resaltar el carácter polisémico de moi como símbolo. Moi, en Saterémawé, puede ser traducido literalmente como serpiente, pero también puede ser usado como alegoría de una de las cualidades de la serpiente, la transformación. Si por un lado, en el caso de moiria, esa transformación aparece como alteración, en otro de los casos de estas mujeres míticas, moi aparece también como principio del orden social. En el caso de Uniawasap, ella fue fecundada por una serpiente, y es por esta relación con moi, que su hijo, el guaraná, tiene la capacidad de transformar las palabras en hechos. Esa cualidad de moi, está asociada a las representaciones de esas mujeres míticas. Cuando le pregunté a los ancianos sobre quienes eran estas mujeres míticas, ellos me respondieron que eran hermanas. La categoría hermanas no puede tomarse solo en el sentido descriptivo, una vez que por el sistema clasificatorio puede ser aplicado a todas las mujeres de un ywania.

La mitología Sateré-Mawé está marcada por narrativas que transforman los parientes por afinidad en enemigos. En la leyenda del halcón real, este lucha contra los parientes de la madre, que eran del ywania de los peces, de los animales de agua. En el mito del origen del agua, los $i^{\prime} k w y t(\mathrm{MB})$ envenenan al personaje del mito. En el mito del guaraná, son los hermanos de la madre, $i^{\prime} k w y t(\mathrm{MB})$ que matan al personaje que se transformará en la planta de guaraná. Como en otros grupos amazónicos, la relación de afinidad, proyectada al plano de la cosmología, presenta a los parientes por afinidad como enemigos (Overing Kaplan 1984; Viveiros de Castro 2002: 168ss). 
En este cuerpo de mitos, marcado por el conflicto con los i'kwyt, debemos destacar uno, marcado por la ausencia de esta relación. En el mito de mypynukuri, los seres míticos aparecen como donadores de mujeres y es en este mito que se explica, también el origen del ritual de la tucandira. La narrativa de este mito se centra en la figura de mypynukuri, un tatú-açú, un armadillo grande, (un peludo), que saco de la tierra las haryporia/mujer y se las entregó a su hermano menor. Este por su vez, las donó para los Sateré-Mawé. Desde este punto de vista mypynukuri se transforma en el donador mítico de mujeres, y transforma a los hombres, en particular a los que participan del ritual, en receptores de mujeres. Este es uno de los índices que relacionan el ritual con la transformación de eses afines virtuales, que pueden ser efectivados.

Durante uno de los viajes de campo, cuando volví a la localidad en la que había presenciado por primera vez el waumat, encontré al cantador que dirigió el ritual. Al ser consultado sobre a posibilidad de volver a realizar el ritual, para registrarlo, el cantador me dijo que para hacerlo bien, habría que convidar a los parientes que viven río arriba. A la luz del análisis teórico, por ser un grupo patrilineal y patrilocal, eses parientes deberían ser los parientes por afinidad, los i'kwyt. Esa hipótesis fue confirmada durante mi último viaje de campo, cuando mi informante concordó con mi raciocinio y completó: "Los i'kywyt son los primeros a colocar la mano en el guante con hormigas. La fiesta de la tucandira es hecha para los i'kwytyt.

Volvamos al relato de Nunes Pereira:

"Este, entonces baila y canta, si es que se puede llamar de danza a esas muecas y saltos, berros y gritos, dados con la cara alegre. Así recorren el espacio del círculo abierto, entre los aplausos de la tribu hasta que, siendo soltero, alguna mujer se compadezca de él y rompiendo el círculo vaya a tirarle los guantes o entonces, que el tuxaua juzgue suficiente la prueba y el mismo se los saque. Entonces el tuxaua hace sonar el cotecá, la multitud se levanta y sigue para el frente de otra casa, donde paran y se repite nuevamente la danza, siendo otro el paciente, quedando atrás, el elegido y su protectora, o tirado en una red, curtiendo su sufrimiento, sí ninguna mujer se agradó por él.

Cabe destacar aquí que la mordida de la tucandira produce efectos afrodisíacos y que la unión inmediata de los sexos hace pasar los dolores y malos efectos. Sí el que sufre el martirio se casó en pruebas anteriores, es la mujer quien le retira los guantes en las otras pruebas que pasa. De aquí nace el error que circula, 
de que estas pruebas son para casamiento. Sí así fuese, los quivus, esto es, los viudos, no pasarían más por ninguna prueba, cuando, en tanto, mismo después de elegidos, tienen que pasar por siete pruebas (Nunes Pereira 2003: 66).

En el parágrafo citado, Nunes Pereira hace importantes revelaciones, condimentadas por un aire de ingenuidad, producto de la falta de formación académica como antropólogo, y del componente erótico que acompañaba el imaginario exótico. No podemos llevar en serio los argumentos analgésico-sexuales, ni que sea compasión lo que lleva a las jóvenes a unirse a los muchachos.

El ritual de la tucandira guarda analogías con el ritual del cauin de los guerreros Araweté, descripto por Viveiros de Castro (1986, 2006: 26ss), tanto en los cantos de guerra, como en la purificación. Viveiros de Castro, en los Araweté, enfatiza la transformación del guerrero con la incorporación del enemigo muerto. En el caso de la Tucandira, la explicación de la purificación esta relacionado con la haryporia. Como afirmó uno de los painis, la tucandira entra en la gente por medio de las aguijoneadas, por eso es necesaria la purificación por medio de vómitos y escoriaciones en las piernas. En caso de que no se complete la purificación, la haryporia/tucandira se lleva al hombre, que muere. Analíticamente, ese movimiento de incorporación de la tucandira/haryporia, y posterior purificación no puede ser interpretado como matrimonio, mas puede si ser interpretado como la transformación performática de estos afines potenciales en afines efectivos (Viveiros de Castro 2002: 87ss).

Hay un dato que me llamó la atención. Una de las veces que asistí al ritual, una de las jóvenes, hija del cantador, colocó la mano en el guante con hormigas. Cuando le pregunté al cantador, él me respondió que el hombre que se case con su hija va tener que haber colocado la mano en el guante más veces que ella. O sea, el ritual ocupa un lugar central en la efectivación de la afinidad. No se trata de un ritual de casamiento, mas de un ritual de casabilidad, que transforma los jóvenes afines potenciales en cónyugues potenciales para las mujeres.

En el caso del parentesco Sateré-Mawé, el ritual de la Tucandira puede tener un papel análogo al del casamiento prescriptivo entre primos cruzados en LevíStrauss (1982). En el esquema de Leví-Strauss (1967), esas estructuras elementales impulsaban la circulación de mujeres, en sistemas donde circulan cuerpos, palabras y bienes. Desde ese prisma estructuralista, el parentesco podía ser visto como un aparato comunicacional. Por otro lado, el ritual es un poderoso 
aparato comunicacional (Leach 1966, 1995; Wolf 1999) y como tal ejerce un poder que se sobrepone a otros tipos de comunicación (Alvarez 2000).

La transformación ritual de afines potenciales en afines efectivos, en un ritual realizado con afines, no puede dejar de tener una influencia en la circulación de mujeres y en la reproducción de alianzas entre ywanias. Ese ritual tiene el poder de crear estructuras análogas a las estructuras elementales de Leví-Strauss y al dravidianato de Dumont. Llegamos a un punto en que no podemos entender el parentesco Sateré-Mawé sí no consideramos el poder estructural del ritual.

"Barbosa Rodrigues, describiendo, en el Tratado de Emancipación de los Maués, la fiesta Veaperiá, pretende que, anualmente, al ser ella realizada, a la orden del jefe de la maloca, y en presencia de los padres, el neófito recibía a su compañera, que de él se compadeciera, asistiendo a la iniciación, de la cual el uso del guante, hirviendo de tucandiras, era la prueba más cruel.

Hoy en día puede ocurrir una de esas uniones, por ocación de la fiesta de la tucandira -quiera inspirado por el sentimiento de piedad, quiera por cualquier otro-, mas no quiere decir que eso sea obligatoriamente respetado por tradición o por convención social o religiosa" (Nunes Pereira, 2003:79).

El autor hace mención a una no obligatoriedad del ritual. A pesar de esto, una de las recurrencias encontradas en el trabajo de campo fué que los no-sateré (sean mestizos o indios) que se casaron con mujeres Sateré-Mawé tuvieron que pasar por el ritual de la tucandira. Pasar por el ritual era la condición para ser aceptados por los parientes de la mujer, para transformarse en $i^{\prime} k w y t$.

Una de las cualidades del dravidianato de Viveiros de Castro (2002), como una torsión del dradiviano de Dumont (1983), fue que el anillo externo estaba contenido por los enemigos. La figura de los enemigos matiag hup aparece constantemente en la cosmología y en la referencia a los otros en general y a los karaiwa (blancos) en particular. El ritual tiene el poder ilocucionario de transformar esos enemigos en afines efectivos, mas no los transforma en consanguíneos, por tanto en Sateré-Mawé. Ellos, sus hijos y sus descendientes permanecerán como un ywania mestizo, viviendo junto a la familia de su mujer. 


\section{Conclusión}

A un nivel general, el trabajo muestra como el parentesco, para la antropología no es sólo parentesco, es teoría antropológica, una teoría construida por diversas tradiciones teóricas. Por otro lado, esas diferentes tradiciones teóricas realzan diversas características al ser aplicadas a un caso concreto, como el de los Sateré-Mawé.

Las diferentes perspectivas teóricas sobre el material Sateré-Mawé revelan la organización formal del sistema de parentesco, sus implicaciones políticas en el ordenamiento de la sociedad y la relevancia de las relaciones de afinidad. Este análisis teórico permite trascender las explicaciones conscientes y los datos de la observación directa para transformarse en una puerta de entrada para la alteridad.

Trabajamos a la luz de diversas tradiciones antropológicas relativas al parentesco. Describimos el sistema con la perspectiva formalista de la tradición norteamericana; exploramos sus implicaciones políticas con la perspectiva británica; y analizamos la alianza a la luz de las estructuras elementales y del dradiviano de Dumont. Posteriormente exploramos la relación del modelo con los trabajos sobre afinidad en las tierras bajas, con especial atención en el modelo del dravidianato que ordena los parientes consaguíneos y por afinidad en circulos concentricos, contenidos por los enemigos. Viveiros de Castro (2002) señala la necesidad lógica de rituales que permitan atravesar esas esferas.

El análisis del ritual de la Tucandira a la luz de las deducciones de las teorías de parentesco permite realizar una nueva interpretación del ritual y revela un pós-dravidiano, en el que el ritual tiene el poder ilocucionario de transformar los afines virtuales en afines efectivos y los enemigos en afines. De nuestro análisis del ritual, desde la perspectiva del parentesco, se desprende que el ritual de la tucandira estructura las relaciones de parentesco. En una sociedad que no practica el casamiento entre primos cruzados, ni un dradiviano en los molde hindúes, el ritual ordena la comunicación de mujeres y reproduce las alianzas entre afines y consanguíneos a lo largo de generaciones, esto es, tiene el mismo poder estructural que las instituciones tradicionales de parentesco, como el casamiento entre primos cruzados. El ritual transforma el dravidianato amazónico en un pós-dradiviano que, como género cruzado, entrelaza consanguíneos y afines, cosmología y parentesco, alianza y descendencia por medio de un poderoso ritual que tiene el poder de transformar la condición del iniciado, de enemigo en afín, de afín potencial en afín 
afectivo. Finalmente este análisis muestra que los limites del parentesco en Amazonas trasciende las instituciones de parentesco y señala la importancia de los rituales para este parentesco pós-dradiviano.

\section{Bibliografia}

ALVArez, Gabriel Omar. 200o. Mercosul ritual. Políticos e diplomatas no processo de Integração do Conesul. Tese de Doutorado. Departamento de Antropologia, Universidade de Brasília.

ALVAREZ, Gabriel Omar. 2002. Amazônia cidadã: previdência social entre as populações tradicionais da Região Norte do Brasil. Brasília: MPAS.

ALVAREZ, Gabriel Omar. 2007. "O Ritual da Tucandeira entre os SateréMawé: aspectos simbólicos do waumat”. In: Câmara Cabral e Rodrigues (org.). Línguas e Culturas Tupi. Campinas: Curt Nimuéndaju; Brasília: Lali/ UnB, p. 59-72.

ALVAREZ, Gabriel Omar. 2010. "Pós-dradiviano Sateré-Mawé: parentesco e rituais de afinabilidade", in Câmara Cabral, Rodrigues, Duarte (orgs.). Línguas e Culturas Tupí, Vol. 2. Campinas, SP: Curt Nimuendajú; Brasília: LALI/UnB, p. 195-220.

AUSTIN, John LLangshaw. 1997. How to do things with words. Cambridge: Harvard University Press.

BALANDIER, Georges. 1977. Antropológicas. São Paulo: Cultrix.

BASSO, Ellen B. 1984. "A husband for his daughter, a wife for her son: strategies for selecting a set of in-laws among the kalapalo". In: K. Kensinger, (org.), Marriage practices in lowland South America. Chicago: University of Illinois Press, p. 33-44.

CARDOSO DE OLIVEIRA, Roberto. 1988. Sobre o pensamento antropológico. Rio de Janeiro: Tempo Brasileiro; Brasília: CNPq.

DUMONT, Louis. 1975. Introducción a dos teorías de la antropología social. Barcelona: Ed. Anagrama.

DUMONT, L. 1983. Affinity as a value. Marriage alliance in South India, with comparative essays on Australia. Chicago: The University of Chicago Press, p. 3-171.

DUMONT, Louis. 1992. Homo Hierarquicus. São Paulo: Editora da Universidade de São Paulo.

FAUSTO, Carlos. 1995. "De primos e sobrinhas: terminologia e aliança entre os 
Parakana (Tupi) do Para”. In: E. Viveiros de Castro, Antropologia do Parentesco: estudos ameríndios. Rio de Janeiro: UFRJ p. 61-120.

FIGUEROA, Alba Lucy Giraldo. 1997. Guerriers de l'écriture et comerçants du monde enchanté: historie, identité et traitement du mal chez les Sateré-Mawé (Amazonie centraal, Brésil). Tese de doutorado. École de Hautes Etudes em Sciences Sociales, Paris.

FERNANDES, Florestan. 1989. A organização social dos Tupinambá. São Paulo: Hucitec; Brasília: Editora da UnB.

LARAIA, Roque de Barros. 1972. Tupi: índios do Brasil atual. São Paulo: FFLCH/USP.

LEACH EDMUND, R. 1966. "Ritualization in man in relation to conceptual and social development". In: Philosophical Transactions of the Royal Society of London. Series B, 772, (251): 403-408.

LEACH EDMUND, R. 1995. Sistemas Políticos da Alta Birmânia. São Paulo: Editora da Universidade de São Paulo.

LEACOCK, Seth. 1964. "Economic life of the Maué Indians”. Boletim do Museu Paraense Emilio Goeldi, Nova Série, Antropologia 19, 30 pp.

LÉVI-STRAUSS, Claude. 1967. Antropologia estrutural. Rio de Janeiro: Tempo Brasileiro.

LÉVI-STRAUSS, Claude. 1982. As estruturas elementares de parentesco. Petrópolis: Vozes.

LORENZ, Sônia da Silva. 1992. Sateré-Mawé: os filhos do guaraná. São Paulo: Centro de Trabalho Indigenista.

MACDONALD, Frederick. 1965. "Some considerations about Tupi-Guarani kinship structures”. In: Boletim do Museu Paraense Emilio Goeldi, , n. 26, 20 pp.

NIMUENDAJÚ, Curt. 1948. “The Maue and Arapium”. In: Steward (Ed.) Handbook of South American Indians, Bulletin 143, vol. 3 The tropical forest tribes. Washington: Smithsonian Institution Bureau of American Ethnology.

PEREIRA, Nunes. 1967. Moronguêtá. Um decameron indígena. Rio de Janeiro: Civilização Brasileira.

PEREIRA, Nunes 2003. Os índios Maués. Manaus: Valer.

OVERING KAPLAN, Joanna. 1984. "Dualism as an expression of differences and danger: marriage exchange and reciprocity among the Piaroa of Venezuela". In: K. Kensinger, Marriage Practices in lowland South America. Chicago: 
University of Illinois Press pp. 127-155.

RIVIÈ RE, Peter. 1969. Marriage among the Trio. A principle of social organization. Oxford: Clarendon Press.

RIVIÈRE, Peter. 2001. O indivíduo e a sociedade na Guiana: um estudo comparativo sobre a organização social ameríndia. São Paulo: Editora da Universidade de São Paulo.

SHAPIRO, Judith. R. 1984. "Marriage rules, marriage exchange and the definition of marriage in lowland south american societies”. In: K. Kensinger, Marriage Practices in lowland South America. Chicago: University of Illinois Press, p. 1-32.

TAMBIAH, Stanley Jeyara. 1985. Culture, thought, and social action. An anthropological perspective. Cambridge, Massachusetts: Harvard University Press.

VAN GENNEP, Arnold. 1978. Os ritos de passagem. Petrópolis: Vozes.

VILAÇA, Aparecida. 1993. "O canibalismo funerário Pakaa-Nova: uma etnografia”. In: E. Viveiros de Castro, M. Carneiro da Cunha, Amazônia: etnologia e história indígena. São Paulo: Núcleo de História Indígena e do Indigenismo da USP; Fapesp, pp. 285-310.

VIVEIROS DE CASTRO, Eduardo. 1986. Araweté: os deuses canibais. Rio de Janeiro: Jorge Zahar Ed.

VIVEIROS DE CASTRO, E. (org.). 1995. Antropologia do parentesco: estudos ameríndios. Rio de Janeiro: UFRJ.

VIVEIROS DE CASTRO, E. 1996. "Ambos os três: sobre algumas distinções tipológicas e seu significado estrutural na teoria do parentesco". Anuário Antropológico/95. Rio de Janeiro: Tempo Brasileiro.

VIVEIROS DE CASTRO, E. 2002. A inconstância da alma selvagem. São Paulo: Cosac \& Naify.

WOLF, Eric, R. 1999. Envisioning Power. Ideologies of dominance and crisis. Berkley: University California Press.

\section{Sobre el autor}

Prof. Dr. Gabriel O. Alvarez, profesor adjunto, DE, sub-coordenador del Programa de Pos-Graduación Antropología Social, Facultad de Ciencias Sociales, Universidad Federal de Goiás, PPGAS/FCS/UFG. Doctor en Antropología Social (2000) por la Universidad de Brasília, PPGAS/DAN/ICS/ 
UnB, Maestría en Antropología (1995) por la Universidad de Brasília, PPGAS/ DAN/ICS/UnB.

Principales libros publicados: Satereria: Tradición y Política Sateré-Mawé (2009); Tradiciones Negras, Políticas Blancas, presion social y poblaciones afro-brasileñas (2006), Industrias Culturales en el Mercosur (org.), (2003) Amazonia Ciudadana, previsión social entre las poblaciones tradicionales de la región Norte de Brasil, (2002). El autor posee 11 artículos publicados en publicaciones periódicas y 9 capítulos en libros. Blog, http//metodoetnografico. blogspot.com.

Dirección para correspondencia: PPGAS/FCS/UFG, Universidad Federal de Goiás, Campus II, FCS - Caixa Postal 131 - CEP: 74.001-970, Goiânia, GO. E-mail: gabriel.o.alvarez@gmail.com Una versión de este articulo, en Portugués fue presentada en el seminario Culturas e Linguas Tupís, Alvarez, 2010. in S. Câmara Cabral, A. Rodrigues, Duarte (orgs.). Línguas e Culturas Tupí, Vol. 2. Campinas, SP: Curt Nimuendajú; Brasília: LALI/UnB.

Received 30/03/11, approved 24/08/11 